\title{
FORMATION OF MEMBRANES BY MEANS OF IMMERSION PRECIPITATION
}

\section{PART II. THE MECHANISM OF FORMATION OF MEMBRANES PREPARED FROM THE SYSTEM CELLULOSE ACETATE-ACETONE-WATER}

\author{
A.J. REUVERS and C.A. SMOLDERS \\ Department of Chemical Technology, Twente University of Technology, P.O. Box 217, 7500 AE \\ Enschede (The Netherlands)
}

(Received June 11, 1986; accepted in revised form March 16, 1987)

\section{Summary}

Using equations and boundary conditions derived in Part I*, calculations have been performed on the ternary diffusion processes that occur in a cellulose acetate (CA)-acetone casting solution immersed into a water bath. The necessary concentration-dependent thermodynamic and hydrodynamic parameters have been derived from experimental data on the three limiting binary mixtures. Calculations show that immersion of the polymer solution into the coagulation bath results in an instantaneous increase of the polymer concentration at the surface of the solution. For a $\mathrm{CA}$-acetone casting solution the thickness of this concentrated surface layer will increase until the onset of liquid-liquid demixing by means of nucleation and growth of the diluted phase that fixes the asymmetric polymer distribution in the film. The moment of onset of the demixing process depends on the thickness of the film. However, addition of a certain minimum amount of water to the casting solution results in an instantaneous onset of liquid-liquid demixing upon immersion, yielding a membrane with a very thin skin layer. The model calculations have been confirmed by light transmission measurements performed on immersed casting solutions.

\section{Introduction}

In Part I of this paper equations and boundary conditions have been derived, describing the diffusion processes that occur after immersion of a cast polymer solution into a nonsolvent bath, prior to possible demixing processes.

In this part this formalism will be applied to a ternary system for which enough parameters are known. The diffusion processes in a polymer solution consisting of cellulose acetate (CA) and acetone immersed into a water bath will be described. This system contains three of the basic components from which Loeb-Sourirajan-type membranes are prepared. Thus, the calculations

*J. Membrane Sci., 34 (1987) 45. 
presented in this paper may serve as a base when considering the mechanism of formation of these type of membranes.

Composition paths will be calculated representing composition changes as a function of time or place for different coagulation bath or casting solution compositions.

Attention will be focussed on the position of the composition path relative to the position of the binodal in the ternary phase diagram. When the composition becomes metastable somewhere in the polymer solution, i.e. when the composition path crosses the binodal curve, nuclei of the polymer-poor phase are formed which may grow out to become the pores in the ultimate membrane.

The moment at which this liquid-liquid demixing process will occur in the casting solution can be calculated with our diffusion model and measured by means of light transmission experiments performed on immersed casting solutions. Thus, a simple method is at hand to test the diffusion model.

Besides the influence of liquid-liquid demixing the influence of aggregate formation on the ultimate membrane structure will also be discussed. Before presenting the values of the parameters used in our model calculations, a short summary of the equations, relations and boundary conditions as derived in Part I will be given.

The equations that describe the ternary diffusion in the polymer solution are given by:

$\frac{\delta\left(\phi_{i} / \phi_{3}\right)}{\delta t}=\frac{\delta}{\delta m}\left\{\sum_{j=1}^{2} \bar{V}_{i} \phi_{3} L_{i j} \frac{\delta \mu_{j}}{\delta m}\right\}, \quad i=1,2$

where $\bar{V}_{i}$ and $\phi_{i}$ are the partial specific volume and the volume fraction of component $i$, respectively; $\mu_{j}$ is the chemical potential of component $j$, and $m$ is a special position coordinate described in Part I. The subscripts 1, 2 and 3 refer to nonsolvent, solvent and polymer, respectively. For a complete list of symbols we refer to Part I.

The ternary coefficients $L_{i j}$ have been expressed as a function of ternary frictional coefficients $R_{i j}$ :

$L_{i j}=\beta_{i j} / \alpha$,

where

$\beta_{12}=\beta_{21}=c_{1} c_{2} R_{12}$

$\beta_{11}=c_{1}\left(c_{1} R_{12}+c_{3} R_{23}\right)$

$\beta_{22}=c_{2}\left(R_{2} R_{12}+c_{3} R_{13}\right)$

and 
$\alpha=c_{3}\left(c_{2} R_{12} R_{23}+c_{1} R_{12} R_{13}+c_{3} R_{13} R_{23}\right)$

Here $c_{i}$ is the concentration of component $i$ in $\left(\mathrm{kg} / \mathrm{m}^{3}\right)$.

The ternary frictional coefficient $R_{12}$ has been related to the mutual diffusion coefficient $D$ and the chemical potential $\mu_{1}$ in the binary solvent-nonsolvent mixture:

$R_{12}\left(\phi_{1}, \phi_{2}\right)=R_{12}\left(\phi_{1}^{\prime}\right)\left(\phi_{3}=0\right)=\frac{\bar{V}_{2} \phi_{1}^{\prime}}{D\left(\phi_{1}^{\prime}\right)} \times \frac{\delta \mu_{1}}{\delta \phi_{1}^{\prime}}$

where $\phi_{1}^{\prime}=\phi_{1} /\left(\phi_{1}+\phi_{2}\right)$.

The ternary frictional coefficient $R_{23}$ has been related to the binary sedimentation coefficient $s_{3}$ :

$R_{23}\left(\phi_{2}, \phi_{3}\right)=R_{23}\left(\phi_{3}\right)\left(\phi_{1}=0\right)=\frac{\left(1-\phi_{3}\right) \bar{V}_{3}\left(\rho \bar{V}_{2}-1\right)}{\phi_{3}} \times \frac{1}{s_{3}\left(\phi_{3}\right)}$

where $\rho$ is the density of the binary polymer-solvent mixture.

The frictional coefficient $R_{13}$ has been related to $R_{23}$ :

$R_{13}=C\left(\bar{V}_{1} / \bar{V}_{2}\right) R_{23}$

$C$ will be assumed to be a constant that has to be estimated.

The binary diffusion in the coagulation bath is described by the following equation:

$\frac{\delta \phi_{i}}{\delta t}=\frac{\delta}{\delta y}\left\{D\left(\phi_{i}\right) \frac{\delta \phi_{i}}{\delta y}\right\}-\frac{\delta \phi_{i}}{\delta y} \times \frac{\delta X(t)}{\delta t}$

where $y$ is a special position coordinate relative to the moving interface between bath and film, and $X(t)$ is the position of this interface.

The boundary conditions at this interface are as follows:

- The chemical potentials are equal at both sides of the interface for all three components.

- The fluxes of component 1 and those of component 2 are equal at both sides of the interface.

In the next sections the values of the parameters used in the diffusion equations will be given. These parameters are:

- the binary thermodynamic interaction parameters, $g_{12}\left(\phi_{2}\right), g_{23}\left(\phi_{2}\right)$ and $\chi_{13}$ from which the chemical potentials are derived;

- the mutual diffusion coefficient $D\left(\phi_{1}\right)$ for acetone-water mixtures and the sedimentation coefficient $s_{3}\left(\phi_{3}\right)$ for CA in acetone:

- the molecular weight $M_{i}$ and the constant partial volume $\bar{V}_{i}$ for $i=1,2$ and 3. 


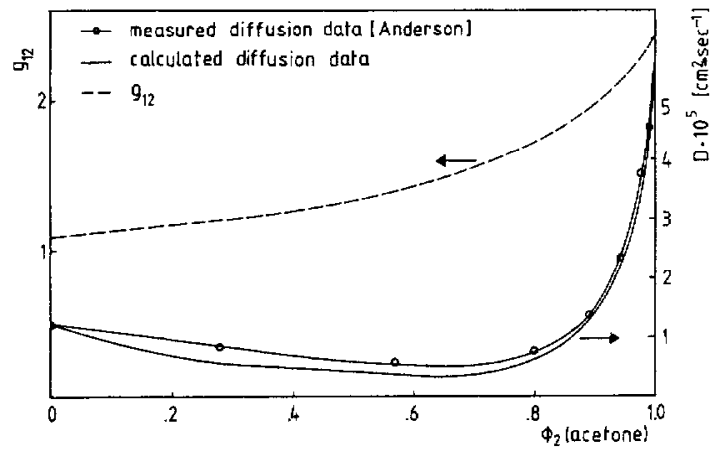

Fig. 1. Concentration dependence of the thermodynamic interaction parameter $g_{12}$ and the mutual diffusion coefficient $D$ for the binary system acetone-water at $25^{\circ} \mathrm{C}$.

Thermodynamic interaction parameters $g_{12}, g_{23}$ and $\chi_{13}$ derived from experimental data

In Appendix A of Part I, expressions for the chemical potentials $\mu_{1}, \mu_{2}$ and $\mu_{3}$ are presented. In this section the values of the thermodynamic interaction parameters will be presented for the binary mixtures acetone-water, $\mathrm{CA}$-acetone and CA-water.

The concentration-dependent parameter $g_{12}\left(\phi_{2}\right)$, representing the interaction between water and acetone, has been calculated from literature data [1] on the excess free energy $G^{\mathrm{E}}$ as follows [2]:

$g_{12}\left(\phi_{2}\right)=\frac{1}{x_{1} \phi_{2}}\left[x_{1} \ln \left(x_{1} / \phi_{1}\right)+x_{2} \ln \left(x_{2} / \phi_{2}\right)+G^{\mathrm{E}} / R T\right]$

where $x_{1}$ represents the mole fraction of water in the mixture.

The calculated values for $g_{12}$ are strongly concentration dependent as can be seen in Fig. 1. The parameter $g_{12}\left(\phi_{2}\right)$ for acetone-water at $25^{\circ} \mathrm{C}$ can be described as follows:

$g_{12}=0.979+1.127 \exp \left(-2.306 \phi_{1}\right)+0.292 \exp \left(-12.564 \phi_{1}\right)$

The concentration-dependent parameter $g_{23}$ for $\mathrm{CA}$-acetone mixtures, determined by means of osmotic pressure measurements, has been given by Altena [3]:

$g_{23}=0.645-0.11 \phi_{2} \quad\left(25^{\circ} \mathrm{C}\right)$

$g_{23}$ appears to be less strongly concentration dependent than $g_{12}$.

The thermodynamic interaction between water and CA is described by means of a constant parameter $\chi_{13}$, measured from swelling experiments [4]:

$\chi_{13}=1.4 \quad\left(25^{\circ} \mathrm{C}\right)$ 


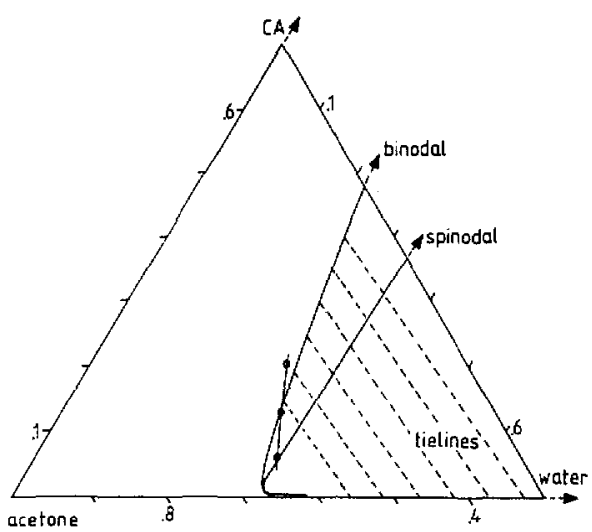

Fig. 2. Isothermal phase diagram for the system $\mathrm{CA}$ /acetone/water. - - : measured position of the liquid-liquid demixing gap at $20^{\circ} \mathrm{C}$ [5]. The binodal and the spinodal are calculated using interaction parameters measured at $25^{\circ} \mathrm{C}$.

These binary parameters will be used in the expressions for the chemical potentials of the ternary system $\mathrm{CA}$-acetone-water.

\section{The liquid-liquid demixing gap}

The ternary system CA-acetone-water is not miscible in all proportions. It is possible to calculate the binodal and spinodal curves, using the expressions for the chemical potentials described in Appendix A of Part I and the thermodynamic interaction parameters described in the previous section. We have calculated the binodal and spinodal curve assuming the CA to be monodisperse, with $M_{3}$ equal to 27,000 .

In Fig. 2 the calculated binodal and tie lines are shown in a phase diagram together with the measured position of the liquid-liquid demixing gap [5]. It can be concluded that the measured position of the demixing gap and the calculated position of the binodal curve are in good agreement. This implies that the chemical potentials in this ternary system are well described by expressions with binary concentration-dependent interaction parameters. These expressions for the chemical potentials are used not only for the calculation of the position of the demixing gap, but also in the diffusion equations (1) and for the calculation of the interfacial boundary compositions.

As shown in Fig. 2, the ternary phase diagram will always be represented partially in this paper.

\section{The binary frictional coefficient $R_{12}$ derived from experimental diffusion data}

In the introduction of this paper the relation between $R_{12}$ and the mutual diffusion coefficient for water-acetone mixtures was given by eqn. (3): 
$R_{12}\left(\phi_{1}\right)=\frac{\bar{V}_{2} \phi_{1}}{D\left(\phi_{1}\right)} \times \frac{\delta \mu_{1}}{\delta \phi_{1}} \quad\left(\phi_{3}=0\right)$

The chemical potential $\mu_{1}$ (per kilogram of component 1 ) is given by:

$\frac{M_{1}}{R T} \times \Delta \mu_{1}=\ln \phi_{1}+\left(1-\phi_{1}\right)\left\{1-s+\left(1-\phi_{1}\right)\left(g_{12}+\phi_{1} \frac{\mathrm{d} g_{12}}{\mathrm{~d} \phi_{1}}\right)\right\}$,

where $s$ is equal to $\bar{V}_{1} M_{1} / \bar{V}_{2} M_{2}$. The interaction parameter $g_{12}$ is given by expression (8).

For mixtures of acetone and water $R_{12}$ is assumed to be independent of $\phi_{1} /\left(\phi_{1}+\phi_{2}\right)$ and to be equal to $R_{12}\left(\phi_{1}=1\right)$ in our model calculations. From equations (3) and (10) it can be derived that

$R_{12}\left(\phi_{1}\right)=R_{12}\left(\phi_{1}=1\right)=\frac{\bar{V}_{1} R T}{M_{2} D\left(\phi_{1}=1\right)}$

As a consequence of the assumption that $R_{12}$ is constant, $D$ must be given by:

$D\left(\phi_{1}\right)=\frac{\bar{V}_{2} M_{2} \phi_{1}}{\bar{V}_{1} R T} \frac{\delta \mu_{1}}{\delta \phi_{1}} \times D\left(\phi_{1}=1\right)$

The diffusion coefficients calculated according to equation (12) will be compared with the measured values presented by Anderson et al. [6] . Both measured and calculated values of $D\left(\phi_{1}\right)$ for the system acetone-water at $25^{\circ} \mathrm{C}$ are shown in Fig. 1. The calculated diffusion coefficients approximately correspond to the measured diffusion coefficients. Hence it can be concluded that expression (11) is a reasonable approximation for $R_{12}\left(\phi_{1}\right)$. From Fig. 1 it follows that $D\left(\phi_{1}=1\right)=1.25 \times 10^{-9} \mathrm{~m}^{2} / \mathrm{sec}$.

Expression (12) will be used in the equation that describes the diffusion process in the coagulation bath.

\section{The binary frictional coefficient $\boldsymbol{R}_{23}$ derived from sedimentation measurements}

The relation between $R_{23}$ and the sedimentation coefficient $s_{3}\left(\phi_{3}\right)$ is given by eqn. (4):

$R_{23}\left(\phi_{3}\right)=\frac{\left(1-\phi_{3}\right) \bar{V}_{3}\left(\rho \bar{V}_{2}-1\right)}{\phi_{3}} \times \frac{1}{s_{3}\left(\phi_{3}\right)} \quad\left(\phi_{1}=0\right)$

Sedimentation experiments have been performed for solutions of CA in acetone in order to obtain values for $R_{23}$ as a function of $\phi_{3}$. The experiments have been performed with a Beckman-Spinco Model E analytical ultracentrifuge. 

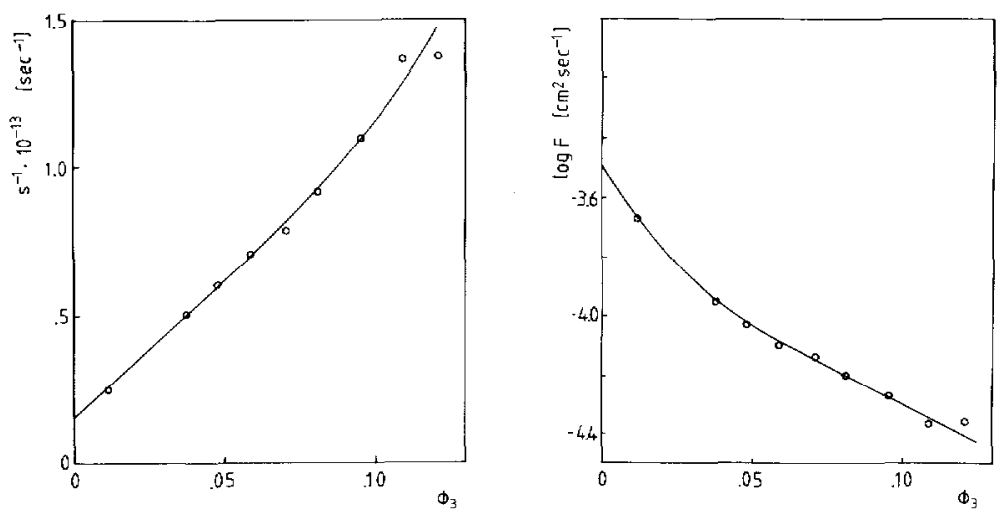

Fig. 3. (a) Concentration dependence of the sedimentation coefficient $s$ for $\mathrm{CA}$ in acetone at $20^{\circ} \mathrm{C}$; (b) concentration dependence of $F$, a variable proportional to the frictional coefficient $R_{23}$ (see eqn. 14).

The sedimentation coefficients have been determined from the displacement of the maximum of the concentration gradient curve.

The CA used, obtained from Eastman Kodak, had an acetyl content of $39.8 \%$ and viscosity number $3\left(\right.$ ASTM) $; M_{\mathrm{N}}=27,000, M_{\mathrm{W}}=54,000$.

The partial volume of $C A$ in acetone has been calculated from the slope of the solution density versus concentration plot. The partial volume appears to be independent of concentration within the experimental concentration range ( up to $\phi_{3}=0.12$ ) and has a value of $0.7 \times 10^{-3} \mathrm{~m}^{3}-\mathrm{kg}^{-1}$ at $20^{\circ} \mathrm{C}$.

In Fig. 3a the sedimentation coefficient is shown as a function of the volume fraction of polymer.

In order to transform expression (4) into a form comparable with expression (11) the variable $F\left(\phi_{3}\right)$ is introduced:

$F\left(\phi_{3}\right)=\frac{R T \phi_{3}}{M_{2}\left(\rho \bar{V}_{2}-1\right)\left(1-\phi_{3}\right)} \times s_{3}\left(\phi_{3}\right)$

When we substitute $F\left(\phi_{3}\right)$ into expression (4), $R_{23}$ is given by:

$R_{23}\left(\phi_{3}\right)=\frac{\bar{V}_{3} R T}{M_{2} F\left(\phi_{3}\right)}$

Thus $R_{12}$ can be compared with $R_{23}\left(\phi_{3}\right)$ by comparing $D\left(\phi_{1}=1\right)$ with $F\left(\phi_{3}\right)$. From Fig. 3b it follows that $F\left(\phi_{3}\right)$ depends strongly on concentration. A linear relationship between $\log F\left(\phi_{3}\right)$ and $\phi_{3}$ exists for $0.05<\phi_{3}<0.12$. Therefore, $F\left(\phi_{3}\right)$ will be approximated for $\phi_{3}>0.05$ by the following expression:

$F\left(\phi_{3}\right)=1.66 \times 10^{-\left(8-5.17 \phi_{3}\right)} \quad\left[\mathrm{m}^{2} / \mathrm{sec}\right]$

For $\phi_{3} \rightarrow 1, F\left(\phi_{3}\right)=1.1 \times 10^{-13} \mathrm{~m}^{2} / \mathrm{sec}$ according to this expression. It can be 
shown that in this limiting case $F\left(\phi_{3}\right)$ must be equal to the self-diffusion coefficient of acetone in CA. Park [7] has measured the self-diffusion coefficient of acetone in CA for $0.15<\phi_{2}<0.25$. Extrapolation of the data of Park to $\phi_{2} \rightarrow 0$ yields a self-diffusion coefficient of $2 \times 10^{-14} \mathrm{~m}^{2} / \mathrm{sec}$. This value is approximately in accordance with the value of $F\left(\phi_{3}=1\right)$. The validity of expression (15) is hereby confirmed.

\section{Influence of the various frictional coefficients on the rate of diffusion}

Knowing the experimental values for the binary frictional coefficients, it is interesting to examine whether the frictional force between solvent and nonsolvent can be neglected compared with the frictional force exerted by the polymer on the low-molecular-weight components as proposed by Cohen et al. [8].

The obscure influence of the various binary frictional coefficients on the rate of diffusion can be elucidated when the following simplifications concerning the thermodynamics of the ternary system are adopted:

$g_{12}=g_{23}=\chi_{13}=0$

$\bar{V}_{1} M_{1}=\bar{V}_{2} M_{2}$

Moreover it is assumed that $R_{13}=\left(\bar{V}_{1} / \bar{V}_{2}\right) R_{23}$ (the constant $C$ in expression (5) is taken to be 1 ).

Consider a ternary solution with gradients in the solvent and nonsolvent concentrations, but without a gradient in the polymer volume fraction. The above-mentioned assumptions make it possible to describe the diffusion process in this solution with the following equation, derived from eqn. (1):

$\frac{\delta \phi_{i}}{\delta t}=\frac{\delta}{\delta x}\left\{\frac{F\left(\phi_{3}\right) D\left(\phi_{1}=1\right)}{\left(1-\phi_{3}\right) F\left(\phi_{3}\right)+\phi_{3} D\left(\phi_{1}=1\right)}\right\} \frac{\delta \phi_{i}}{\delta x}, \quad i=1,2$

This equation describes a kind of binary diffusion process in a polymer matrix of which the density can be chosen. The diffusion coefficient for this process, $D^{\prime}$, is given by the term between brackets. $D\left(\phi_{1}=1\right)=1.25 \times 10^{-9} \mathrm{~m}^{2} / \mathrm{sec}$ and $F\left(\phi_{3}\right)$ is represented by expression (15).

The value of $D^{\prime}\left(\phi_{3}\right)$ will be calculated for $\phi_{3}=0.15$. This polymer volume fraction is approximately equal to a CA weight fraction in acetone of 0.25 , which is the usual CA content of a Loeb-Sourirajan-type casting solution. For $\phi_{3}=0.15$ it follows that $D^{\prime}=1.36 \times 10^{-9} \mathrm{~m}^{2} / \mathrm{sec}$.

When the frictional force acting between solvent and nonsolvent is neglected $\left(D\left(\phi_{1}=1\right) \rightarrow \infty\right)$, as proposed by Cohen et al. [8], it follows that $D^{\prime}=18.6 \times 10^{-9}$ $\mathrm{m}^{2} / \mathrm{sec}$.

When the frictional forces acting between polymer and the low molecular 
weight components are neglected $(F \rightarrow \infty)$ it follows that $D^{\prime}=1.47 \times 10^{-9}$ $\mathrm{m}^{2} / \mathrm{sec}$.

From these results it can be concluded that for the system $\mathrm{CA}$-acetone-water the frictional force acting between solvent and nonsolvent may not be neglected (as proposed by Cohen et al. [8]) if one wishes to describe the diffusion behavior at a polymer volume fraction of 0.15 .

\section{Calculated composition paths valid for a short period after immersion}

In this section will be presented the calculated composition changes in an immersed CA solution during the period over which the bottom composition of the film remains unchanged. In Part I we have shown that during this period the interfacial boundary compositions remain constant.

First, a short description will be given of the iterative procedure followed for the calculation of concentration profiles in the film as a function of time, for certain initial compositions of the coagulation bath and the polymer solution:

- The diffusion equations for the semi-infinitely thick film (eqn. (1), Part II) are solved for a randomly chosen interfacial boundary composition which is situated on the binodal in the phase diagram.

- The fluxes of solvent and nonsolvent at the interfacial boundary of the polymer solution are calculated with eqn. (25) of Part I.

- These fluxes are used to calculate the rate of movement of the interfacial boundary, $\delta X / \delta t$ (eqn. (26), Part I).

- $\delta X / \delta t$ is used to solve the diffusion equation for the coagulation bath (eqn. (6), Part II) for the interfacial boundary composition that is in equilibrium (connected through a tie line) with the interfacial boundary composition of the film.

- The solvent flux at the interfacial boundary of the bath is calculated with eqn. (28) of Part I and compared with the solvent flux at the other side of the boundary, calculated before.

- This procedure is repeated by choosing other interfacial boundary compositions until the calculated solvent fluxes at both sides of the interface are equal.

By changing the interfacial boundary compositions into the direction of the critical point, the calculated solvent flux decreases at the film side of the interface whereas it increases at the bath side of the interface. If the interfacial boundary compositions are situated at the critical point and the calculated solvent flux at the film side of the interface is still higher than the calculated solvent flux at the bath side of the interface, an interfacial boundary will not be formed. This means that a concentration profile will be formed as shown in Fig. $2 \mathrm{~b}$ of Part I of this paper.

In order to examine whether metastable compositions are reached somewhere in the polymer solution, the calculated composition changes in the film 
will be represented in a ternary phase diagram. This can be carried out by representing the composition changes by means of 'composition paths'.

In this section we consider the film to be infinitely thick. Under these circumstances the composition change in the film is a function of $m t^{-1 / 2}$ and the composition path can be interpreted in two different ways:

- The path shows the succession of compositions at a fixed position in the

film from the initial composition to the composition for $t \rightarrow \infty$.

- The path shows the succession of compositions at a fixed moment from the bottom of the film to the interfacial boundary.

The derivation of the composition paths from the calculated concentration profiles is accompanied by a loss of information about the concentration distribution in the film as a function of time. However, in a following section a calculated composition profile will be shown.

For the calculations it is assumed that the initial polymer volume fraction in the film is equal to 0.1 . This corresponds with a CA weight fraction in acetone equal to 0.17 .

In Fig. 4 composition paths are shown for different initial compositions of the coagulation bath, calculated using two different expressions for the unknown parameter $R_{13}$. Which path belongs to which initial concentration follows from Fig. 5, where the boundary composition of the film is shown as a function of the initial composition of the coagulation bath. The corresponding boundary compositions are equally indexed in both figures. The tie lines in Fig. 4 connect the interfacial boundary compositions of the film and the coagulation bath.

An increase of the initial acetone concentration in the bath is accompanied by a decrease of the polymer concentration at the interfacial boundary of the film. At a certain initial solvent concentration in the bath the two-phase system can no longer exist and the interfacial boundary layer will disappear. This means that the polymer starts to dissolve in the coagulation bath.

However, the polymer will also dissolve in the coagulation bath if this contains exactly (or a little more than) the amount of solvent necessary to create an interfacial boundary layer. For example, if the polymer concentration at the boundary of the film is equal to $5 \%$, the polymer concentration at the boundary of the bath will be $0.1 \%$, which means transfer of polymer from the film. In a following paper we will show that this concentration is in fact considerably higher than $0.1 \%$ because of the polydispersity of the polymer.

At this point, the case will be considered in which the coagulation bath consists of pure water at the start of each experiment. A varying amount of water is added to the casting solution while the polymer volume fraction is kept constant at 0.1 .

In Fig. 6 composition paths are shown which describe the composition change in the film during the time the bottom composition of the film remains 

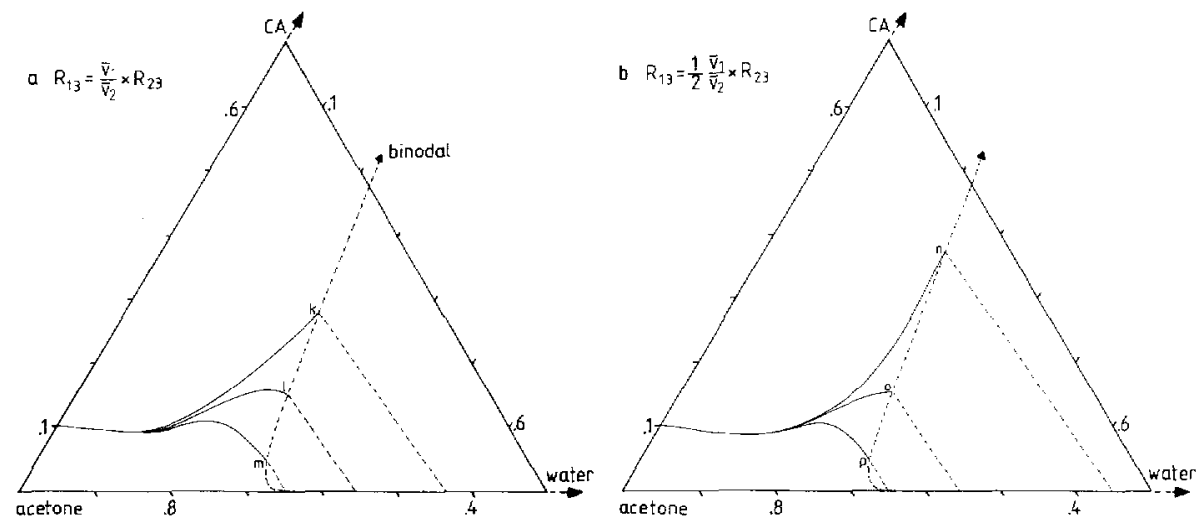

Fig. 4. Calculated composition paths for 10 vol.\% CA solutions immersed into coagulation baths with different initial compositions. The index at the interfacial boundary composition refers to the corresponding initial bath concentration shown in Fig. 5 .

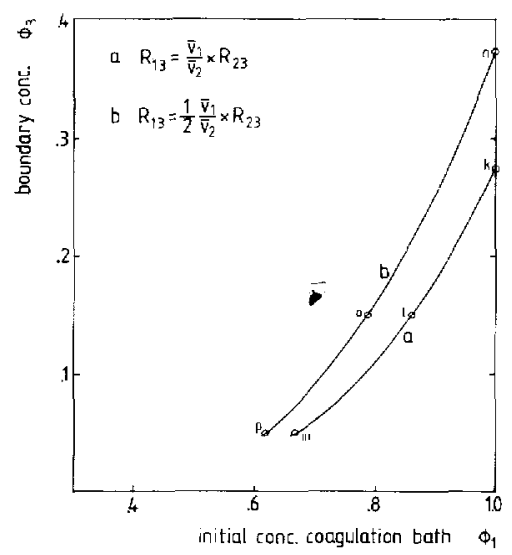

Fig. 5. Polymer concentration at the interfacial bcundary in the immersed film as a function of the initial water concentration in the coagulation bath. The indices at the different boundary compositions refer to the corresponding composition paths shown in Fig. 4.

unchanged. The unknown parameter $R_{13}$ has been varied in the same way as in Figs. 4 and 5.

Figure 6 shows that the polymer concentration at the interfacial boundary increases with increasing water content of the casting solution. However, most striking is the fact that an increasing water content of the casting solution causes the composition path to shift towards the binodal. At a certain water content of the casting solution the composition path even crosses the binodal, which means that immediately after immersion of the film into the bath a metastable composition is reached just beneath the interfacial boundary in the film. 

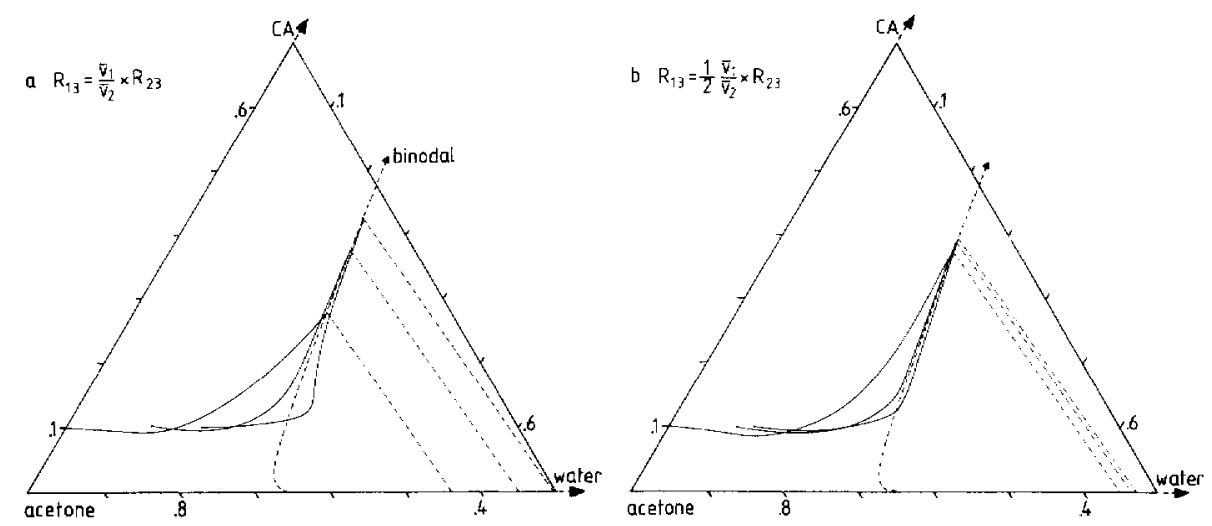

Fig. 6. Calculated composition paths for films of 10 vol. $\%$ CA solutions immersed into pure water coagulation baths, for different water/acetone ratios in the casting solution: (a) $\phi_{1} / \phi_{2}=0 / 100$, $12.5 / 87.5,20 / 80$; (b) $\phi_{1} / \phi_{2}=0 / 100,10 / 90,12.5 / 87.5$.

Previously quenching experiments have been performed in our laboratory on metastable solutions with a degree of supersaturation of about $0.1 \%$ water. These experiments yielded induction times for liquid-liquid demixing which were so short that they could not be detected experimentally. Therefore, it is assumed that liquid-liquid demixing by means of nucleation and growth of the diluted phase takes place immediately after entering the metastable composition region.

When the composition path does not cross the binodal liquid-liquid demixing can not take place; this situation persists at least as long as the composition at the bottom of the film remains unchanged.

Thus the time it takes before liquid-liquid demixing occurs somewhere in the immersed film will decrease from a measurable period $\Delta t$, at zero water content, to zero upon adding a certain critical amount of water to the casting solution. According to our calculations (see Fig. 6) this will happen at a water/ acetone ratio:

$\frac{12.5}{87.5}<\frac{\phi_{1}}{\phi_{2}}<\frac{20}{80}$ for $R_{13}=\left(\bar{V}_{1} / \bar{V}_{2}\right) R_{23}$

$\frac{10}{90}<\frac{\phi_{1}}{\phi_{2}}<\frac{12.5}{87.5}$ for $R_{13}=0.5\left(\bar{V}_{1} / \bar{V}_{2}\right) R_{23}$

In the next section will be shown how this critical value of $\phi_{1} / \phi_{2}$ can be determined experimentally. Comparison of this measured value of $\phi_{1} / \phi_{2}$ with the calculated value of $\phi_{1} / \phi_{2}$ for different expressions for $R_{13}$ enables us to obtain a satisfactory expression for $R_{13}$. 


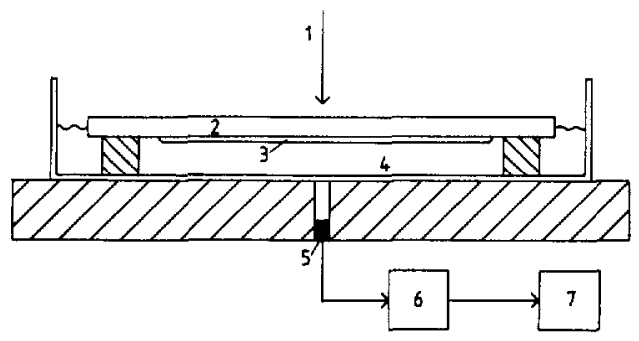

Fig. 7. Measurement of the light transmission through an immersed polymer solution; 1 , light source; 2 , glass plate; 3 , polymer solution; 4 , coagulation bath; 5 , detector; 6 , amplifier; 7 , recorder.

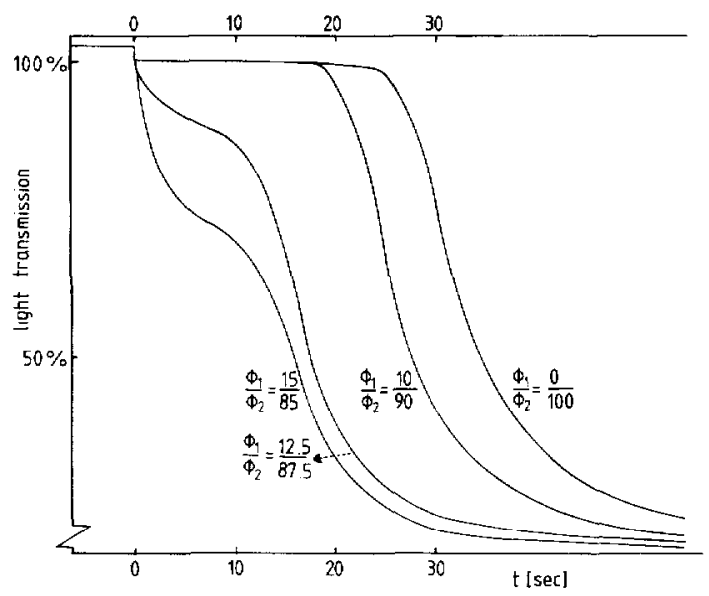

Fig. 8. Percentual light transmission through 10 vol.\% CA solutions immersed into a pure water bath for different water/acetone ratios in the casting solution at $25^{\circ} \mathrm{C}$. At $t=0$ the film and the bath are contacted with each other; initial film thickness: $220 \mu \mathrm{m}$.

\section{Light transmission experiments}

Light transmission experiments on immersed casting solutions have been performed in order to measure the time it takes before liquid-liquid demixing occurs somewhere in the film.

The experimental setup for these measurements is shown in Fig. 7. The membrane is cast on a small glass plate. The glass plate is turned upside down and placed on four points of support in a transparent water bath as quickly as possible. The water level in the bath is just in between the top and bottom of the glass plate to prevent troublesome light scattering at the water surface.

The cast film must be positioned at the lower side of the glass plate for the system CA-acetone-water, because this geometry prevents convection in the coagulation bath as a result of the large density difference between acetone 
and water. Recall the wish to compare the results of these measurements with model calculations where only mass transfer by diffusion is considered.

Above the coagulation bath, a simple desk lamp acts as a light source. After immersion of the cast film in the coagulation bath, the light transmittance is measured and recorded as a function of time. The appearance of optical inhomogenities in the film, as a result of liquid-liquid demixing, causes the light transmittance to decrease.

After the coagulation process a small piece of membrane with a certain surface area, positioned above the detector, is cut out, dried and weighed. In combination with the known volume fraction of polymer in the cast solution the thickness of the immersed film at $t=0$ can be calculated.

These measurements were performed on CA casting solutions containing a variable amount of water and a constant volume fraction of polymer $\left(\phi_{3}=0.1\right)$. We were especially interested in the critical value of $\phi_{1} / \phi_{2}$ in the casting solution where a transition exists from delayed onset of liquid-liquid demixing to instantaneous onset of liquid-liquid demixing. The onset of liquid-liquid demixing in the immersed film is accompanied by a strong decrease in light transmittance.

Experimental results for films with an initial thickness of $220 \mu \mathrm{m}$ are shown in Fig. 8. The onset of liquid-liquid demixing is instantaneous for a water/acetone ratio in the casting solution equal to or larger than $12.5 / 87.5$, while it is delayed for $\phi_{1} / \phi_{2}$ equal to or smaller than $10 / 90$. Consequently, the critical value of $\phi_{1} / \phi_{2}$ lies in between $10 / 90$ and $12.5 / 87.5$.

When this result is compared with the critical values of $\phi_{1} / \phi_{2}$ calculated in the previous section for two different expressions for $R_{13}$, it can be concluded that the experimental critical value of $\phi_{1} / \phi_{2}$ agrees with the critical value of $\phi_{1} / \phi_{2}$ calculated with $R_{13}=0.5\left(\bar{V}_{1} / \bar{V}_{2}\right) R_{23}$.

The measurements performed on casting solutions with a water/acetone ratio smaller than the critical value have been repeated for different film thicknesses. It has been observed that the delay time for the onset of liquid-liquid demixing is proportional to the square of the initial film thickness.

This observed relation confirms the validity of the diffusion model because it can also be derived from eqns. (16), (19), (21), (22) and (23) given in Part I of this paper, by introducing the new dimensionless position coordinates $m / M$ in eqn. (16) and (23) and $y / M$ in eqn. (19) ( $m$ and $y$ are position coordinates defined in Part $\mathrm{I} ; M$, also defined in Part $\mathrm{I}$, is proportional to the initial film thickness).

These new position coordinates yield boundary conditions and diffusion equations in which the geometry of the diffusion problem is only expressed by the factor $1 / M^{2}$, positioned before the right-hand sided terms in eqns. (16) and (19). This means that, relative to the new position coordinates, the rate of diffusion in the coagulation bath and in the film is inversely proportional to the square of the initial film thickness. 
Suppose that a saturation composition is reached first at position $(m / M)^{*}$. Then, the time required for position $(m / M)^{*}$ to reach the saturation composition is proportional to the square of the initial film thickness.

In the previous section composition paths were calculated which describe composition changes that occur during the time the bottom composition of the film remains unchanged. According to our model the composition path in a ternary phase diagram does not shift during this period. As soon as the bottom composition of the film starts to change, however, the composition path in the ternary phase diagram starts to shift and it may cross the binodal after a certain time, according to the observations presented in this section. In the next section calculated composition paths will be presented which approximately describe the composition changes that occur during a longer period of immersion of a film with an initial thickness of $200 \mu \mathrm{m}$. The model calculations will be compared with the observation that the delay time for the onset of liquid-liquid demixing is equal to 20 seconds for an initially $200 \mu \mathrm{m}$ thick, waterfree film immersed into a pure water bath.

\section{Calculated composition paths approximately valid for a longer period of immersion}

After a certain period of immersion of a film with finite thickness the bottom composition of the film will change. As soon as this happens it cannot be argued anymore that the interfacial boundary composition of the film remains constant.

Considering the final result of the precipitation process it is clear that the polymer concentration at the interface will increase. Depicted in a ternary diagram, the boundary composition will shift upwards along the binodal until no solvent is left. The change of composition at the interface is a severe complication of the calculation procedure.

We will approximate the change of the composition in the film for a longer period of immersion by assuming the interfacial boundary composition to remain constant. The approximation becomes worse of course when the bottom composition in the film differs more from the initial composition.

In Fig. 9 the calculated change of the composition path is shown for an initially $200 \mu \mathrm{m}$ thick water-free film, immersed into a pure water bath. The calculations have been performed using two different expressions for $R_{13}$.

With $R_{13}=\left(\bar{V}_{1} / \bar{V}_{2}\right) R_{233}$ the composition path enters the liquid-liquid demixing gap about 40 seconds after immersion of the film. With $R_{13}=0.5\left(\bar{V}_{1} / \bar{V}_{2}\right) R_{23}$ the calculated delay time for the onset of demixing is about 22 seconds. In the previous section it has been shown that light transmission measurements yield a delay time of 20 seconds for the onset of liquid-liquid demixing in an initially $200 \mu \mathrm{m}$ thick film. Again this is in accordance with the calculated result for $R_{13}=0.5\left(\bar{V}_{1} / \bar{V}_{2}\right) R_{23}$. 

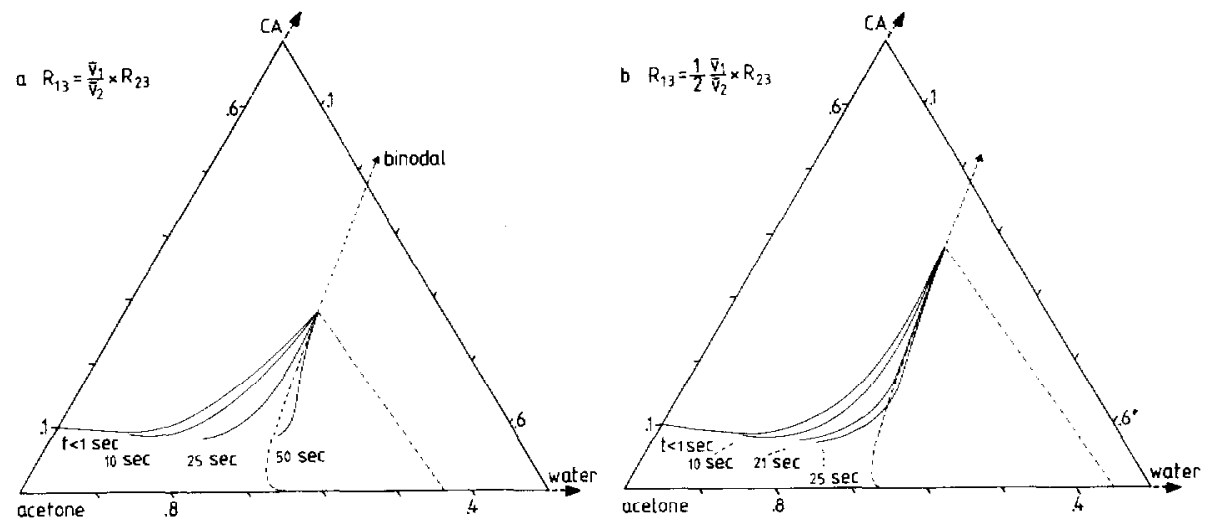

Fig. 9. Approximated change of the composition path at different immersion times for a 10 vol.\% CA solution immersed into a pure water bath; initial film thickness: $200 \mu \mathrm{m}$.

Although the calculated delay time is only an approximation because we assumed the interfacial boundary composition to remain constant, this approximation is confirmed by Fig. $6 \mathrm{~b}$, which shows that the presence of a considerable amount of water in the casting solution hardly influences the interfacial boundary composition.

Thus the light transmission measurements have provided two independent experimental checks which indicate that our diffusion model correctly describes the composition changes in an $\mathrm{CA}$-acetone (-water) solution immersed into a water (-acetone) bath, if $R_{13}$ is chosen to be equal to $0.5\left(\bar{V}_{1} / \bar{V}_{2}\right) R_{23}$. The fact that $R_{13}$ has to be chosen smaller than $\left(\bar{V}_{1} / \bar{V}_{2}\right) R_{23}$ seems reasonable because the smaller water molecules will penetrate the 'polymer network' easier than do the acetone molecules, assuming that in the ternary solution the water molecules do not form clusters like in pure water. (See also the derivation of eqn. (44) in Part I.)

\section{Model calculations in relation to membrane formation and the ultimate membrane structure}

In the previous sections was shown that a reasonable assumption for our model is: $R_{13} \sim 0.5\left(\bar{V}_{1} / \bar{V}_{2}\right) R_{23}$. For this value of $R_{13}$ the actual composition profile is shown in Fig. 10 for an initially water-free film immersed into a pure water bath. The volume fractions of polymer and water are shown as functions of the distance from the interface between the bath and the film $\left(x^{\prime}\right)$. Because the diffusion equations have been solved using the position coordinate $m$, it was necessary to convert the solution using the expression:

$x^{\prime}=\int_{0}^{m} \phi_{3}^{-1}(\xi) \mathrm{d} \xi$ 


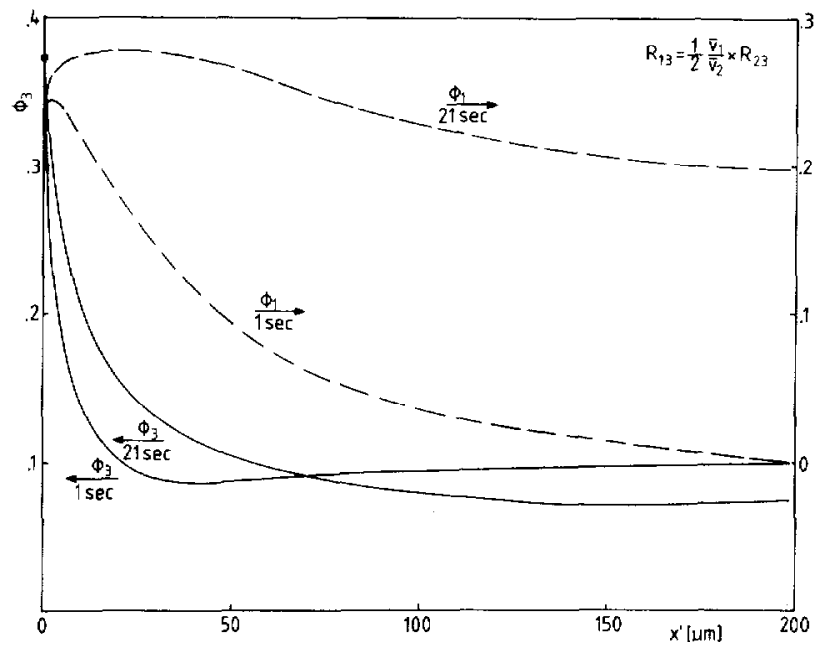

Fig. 10. Calculated composition profiles in a casting solution consisting of $10 \mathrm{vol}$.\% CA in acetone immersed into a pure water bath; $x^{\prime}$ is the distance from the interfacial boundary between the bath and the film; initial film thickness: $200 \mu \mathrm{m}$.

Composition profiles at 1 second and 21 seconds after immersion of the film are shown. These profiles correspond to the composition paths shown in Fig. $9 b$.

The initial film thickness is $200 \mu \mathrm{m}$. According to the calculations, the film thickness hardly decreases during the first 21 seconds after immersion of the film. However, near the interfacial boundary the polymer concentration increased very strongly at the very moment of contact between the polymer solution and the bath. The small region of highly concentrated solution grows out until the onset of liquid-liquid demixing ( 21 seconds after immersion) fixes this asymmetric polymer distribution in the film.

Thus, the asymmetric porosity distribution in the ultimate membrane is determined by the polymer distribution that results from the diffusion processes in the immersed film until the onset of liquid-liquid demixing.

Liquid-liquid demixing by means of nucleation of the diluted phase cannot take place at the interface between the bath and the film because the film composition at the interface remains situated on the binodal during the coagulation process. This is independent of the position of the composition path relative to the position of the binodal in the ternary diagram.

I. When the composition path does not cross the binodal before the bottom composition of the film has changed, which is the case for the ternary system considered in Fig. 10, the region of highly concentrated polymer solution just beneath the interface increases considerably until the onset of liquid-liquid demixing. The delay time for the onset of demixing, and thus the thickness of 
this intermediate layer at the moment of onset of liquid-liquid demixing, depend on the initial position of the composition path relative to the position of the binodal and on the rate of shifting of the composition path towards the binodal in the ternary diagram.

It is interesting that the thickness of the intermediate layer also depends on the initial thickness of the cast film. Because the polymer concentration in this layer is relatively high at the moment of onset of liquid-liquid demixing, the porosity induced by this demixing process will be relatively low while the chance that the ultimate pores will be interconnected is small. Consequently, when liquid-liquid demixing is the only demixing process that is responsible for pores in the ultimate membrane, this intermediate layer will influence the transport behavior of the membrane very strongly. According to this reasoning the transport behavior of this type of membrane is influenced by the thickness of the cast film.

Beneath this effectively dense intermediate layer with a thickness of a few micrometers (see Fig. 10) a thick sublayer is formed which is highly porous because the initial polymer concentration has hardly increased until the onset of liquid-liquid demixing in this layer.

II. When the initial composition path already crosses the binodal, the onset of liquid-liquid demixing will not be delayed and nuclei of the diluted phase will be formed just beneath the top layer in a region where the initial polymer concentration has hardly changed. Consequently, the induced porosity and the interconnectivity of the pores will be high. In a following paper will be shown that the nuclei of the diluted phase formed in this region of the film can even grow out to form macrovoids in the ultimate membrane. The ultimate transport behavior of this type of membranes is only determined by the very thin dense layer formed at the interface between bath and film.

The nuclei of diluted phase, formed beneath the skin layer, cannot grow through the interface between the coagulation bath and the polymer solution film because there exists no condition of supersaturation at the interface. In our opinion a microporous surface layer will only be formed if the formation of an interfacial boundary between bath and film is prohibited by the presence of an excess amount of solvent in the bath (see Figs. 4 and 5). This assumption is in accordance with experimental results of Wijmans et al. [9] concerning the formation of microporous surface layers.

During the immersion process the exchange of solvent and nonsolvent in the demixed film continues, causing an increase of the polymer concentration in the concentrated phase surrounding the pores. When the concentrated phase crosses the glass transition in the isothermal phase diagram the ultimate structure of the membrane is fixed.

Membranes formed by the mechanism of delayed onset of liquid-liquid demixing will be called type I membranes. Membranes formed by the mecha- 
nism of instantaneous onset of liquid-liquid demixing will be called type II membranes.

For type II membranes the thickness of the thin skin layer is very important for the transport properties of the membrane. This thickness is determined by the distance to the interface of the position where the first nuclei of the diluted phase are being formed. Unfortunately our model only predicts that the composition in the film just beneath the interface becomes metastable immediately after immersion of the film. The prediction of the exact position where the first nuclei of the diluted phase are being formed is beyond the scope of this model.

\section{Discussion}

In the previous sections we have considered liquid-liquid demixing by means of nucleation and growth of the diluted phase as the pore-forming mechanism during membrane formation. According to this consideration and our model calculations one can distinguish between two types of membranes:

Type II membranes consisting of two layers: (i) a very thin skin layer where no nucleation of the diluted phase could take place; (ii) a thick sublayer with interconnected pores stretching out to the bottom side of the membrane.

Type I membranes consisting of three layers. In between the two layers mentioned for type II membranes an effectively dense intermediate layer has been formed with a thickness of a few micrometers. Because of the high polymer concentration at the moment of onset of demixing the formation of an interconnective pore structure in this layer has been prohibited.

Type I membranes will be formed only when the initial composition path does not cross the binodal in the ternary phase diagram. We have shown that type I membranes are formed when a CA-acetone solution is immersed into a coagulation bath consisting of water only.

In a following paper will be shown that the type of membrane that will be formed is strongly influenced by the degree of miscibility of the solvent and the nonsolvent. When solvents are used which are better miscible with water than acetone (for example DMSO, DMF or dioxane) type II membranes will be formed.

Besides liquid-liquid-demixing, other porosity-inducing processes like crystallization or aggregate formation may occur during the immersion process. Aggregate formation occurs in several ternary systems $[5,10,11]$ at a certain nonsolvent or polymer concentration. This demixing process, accompanying the gelation process, often results in a nodular or fibrillar structure. In a previous paper [5] we have shown that this process also occurs in CA/acetone/ water solutions, the system discussed in this paper.

We think that aggregate formation will lead to much beter interconnected pores than liquid-liquid demixing (above the critical point) because the solution demixes by means of nucleation and growth of a solid phase. 
Especially in type I membranes the occurrence of this demixing process during the immersion of the cast film will influence the transport properties of the ultimate membrane to a large extent because it will increase the degree of interconnectivity of the pores in the dense intermediate layer beneath the skin layer.

The influence of this demixing process on the structure of the thin skin layer is hard to predict because no experimental data are available on the structure induced by aggregate formation in highly concentrated polymer solutions. The calculations presented in this paper provide the composition range in which these structure analyses should be carried out for the ternary system $\mathrm{CA} /$ acetone/water.

\section{References}

1 R.V. Orye and J.N. Prausnitz, Multicomponent equilibria with the Wilson equation, Ind. Eng. Chem., 57 (5) (1965) 18.

2 F.W. Altena and C.A. Smolders, Calculation of liquid-liquid phase separation in a ternary system of a polymer in a mixture of a solvent and a nonsolvent, Macromolecules, 15 (1982) 1491.

3 F.W. Altena, Phase separation phenomena in cellulose acetate solutions in relation to asymmetric membrane formation, Ph.D. Thesis, Twente University of Technology, Enschede, The Netherlands, 1982, Chap. 6.

4 M.H.V. Mulder, T. Franken and C.A. Smolders, Preferential sorption versus preferential permeability in pervaporation, J. Membrane Sci., 22 (1985) 155.

5 A.J. Reuvers, F.W. Altena and C.A. Smolders, Demixing and gelation behavior of ternary cellulose acetate solutions, J. Polym. Sci., Polym. Phys. Ed., 24 (1986) 793.

6 D.K. Anderson, J.R. Hall and A.L. Babb, Mutual diffusion in non-ideal binary liquid mixtures, J. Phys. Chem., 62 (1958) 404.

7 G.S. Park, Radioactive studies of diffusion in polymer systems. Part 3, Trans. Faraday Soc., 57 (1961) 2314.

8 C. Cohen, G.B. Tanny and S. Prager, Diffusion-controlled formation of porous structures in ternary polymer systems, J. Polym. Sci., Polym. Phys. Ed., 17 (1979) 477.

9 J.G. Wijmans, J.P.B. Baaij and C.A. Smolders, The mechanism of formation of microporous or skinned membranes produced by the immersion precipitation process, J. Membrane Sci., 14 (1983) 263.

10 J.G. Wijmans, H.J.J. Rutten and C.A. Smolders, Phase separation phenomena in solutions of poly (2,6-dimethyl-1,4-phenyleneoxide) in mixtures of trichloroethylene, 1-octanol and methanol: Relationship to membrane formation, J. Polym. Sci., Polym. Phys. Ed., 23 (1985) 1941.

11 A. Labudzinska and A. Ziabicki, Effect of composition and gelation conditions on structural changes accompanying the gelation of PAN, PVA and gelatin solutions, Kolloid Z.Z. Polym., $243(1971) 21$. 